\title{
INFLUENCIA DE LA HIPOXIA SOBRE LA PROFUNDIDAD RADICAL DE Saccharum spp., EN EL VALLE DEL CAUTO, CUBA
}

\section{INFLUENCE OF HYPOXIA ON THE RADICAL DEPTH OF Saccharum spp., IN CAUTO VALLEY, CUBA}

\author{
Sergio Rodríguez¹, Jorge L. Romero², Eduardo Ortega ${ }^{3}$, José Dell`Amico ${ }^{4}$ y Adriano Cabrera ${ }^{5}$ \\ Recibido para evaluación: Abril 1 de 2007 - Aceptado para publicación: Octubre 5 de 2007
}

\begin{abstract}
RESUMEN
En un experimento Ilevado a cabo en un suelo Vertisol del Valle del Río Cauto en Cuba se evaluó el efecto del exceso de agua sobre el desarrollo del sistema radical de seis genotipos de caña de azúcar. Se uso un diseño de parcelas divididas, donde la presencia o no de exceso de agua en el suelo era la parcela y los genotipos eran las subparcelas. Los tratamientos fueron 12 y consistieron en los genotipos C86-12, C86-456, C90-317, C87-51, C1051-73 y C12078, sometidos o no al exceso de agua en el suelo. Se encontró que la Intensidad del Estrés (IE), redujo en un $17 \%$ la profundidad radical en el primer ciclo, mientras que para la primera soca se redujo en un $11 \%$, con marcada diferencia entre genotipos. Los genotipos menos afectados fueron C86-12, C86-456 y C90-317, lo que parece ser un indicativo de estar mejor adaptados para enfrentar procesos de anaerobiosis en el suelo.
\end{abstract}

Palabras clave: Adaptación, caña de azúcar, sobrehumedecimiento.

\begin{abstract}
The effect of water flooding on sugarcane root depth was evaluated in six Cuban sugarcane genotypes planted in a Vertisol at the Rio Cauto Valley. Twelve treatments were planted in a split plot design whit five replications. Two soil water contents were the main plots and six genotypes were the subplot. Sugarcane genotypes were identified as C86-12, C86-456, C90-317, C87-51, C1051-73 y C12078. The results indicated that the Intensity of stress (IE), reduced, on average, root depth by $17 \%$ in the first sugarcane crop cycle and by $11.0 \%$ in the subsequent crop cycles, with marked difference between genotypes. The less affected genotypes were C86-12, C86-456 and C90-317, which seems to be better adapted to anaerobosis.
\end{abstract}

Key Words: Adaptation, sugarcane, flooding.

\footnotetext{
'Universidad de Granma. Carretera vía Manzanillo, km 17. Bayamo CP 85 100. Granma. Cuba. srrguez@udg.co.cu ${ }^{2}$ Corporación Colombiana de Investigaciones Agropecuarias (CORPOICA). Colombia. jorgeromeroferrer@gmail.com

${ }^{3}$ Universidad de la Habana. Cuba

${ }^{4}$ Instituto Nacional de Ciencias Agrícolas. Cuba.

${ }^{5}$ Grupo de Extensión y Servicios Agrícolas. MINAZ. Granma. Cuba.
} 


\section{INTRODUCCIÓN}

Las plantas están distribuidas en ambientes muy diferentes en el globo terrestre, los cuales ofrecen distintas condiciones para o desarrollo vegetal. En ambientes con exceso de agua en el suelo, hay un estrés por baja en la concentración de oxigeno en el medio (Jackson y Drew, 1984), acarreando en una serie de disturbios en el metabolismo de las plantas que se manifiestan por medio de alteraciones en el crecimiento y desarrollo (Kozlowski y Pallardy, 1984; Crane y Davies, 1988). La ausencia parcial (hipoxia) o total (anoxia) de oxigeno afecta la nutrición mineral, la producción y traslocación de reguladores de crecimiento, fotosíntesis, respiración y acumulo de carbohidratos, perjudicando la productividad de varios cultivares comerciales plantados en suelos con bajo drenaje, inundados o encharcados, sujetos a lluvias en períodos prolongados o en los que presentan anaerobiosis localizada, como los compactados. Así, dependiendo de la duración e intensidad del estrés causado por la baja disponibilidad de oxigeno, la planta puede hasta morir (Kawase, 1987).

Muchas especies arbóreas y herbáceas consiguen sobrevivir sobre condiciones de baja disponibilidad de oxigeno durante el estado de crecimiento vegetativo, en cuanto otras mueren en las primeras semanas de inundación de los suelos (Hall y Smith, 1955; Crawford y Tyler, 1969). El grado de tolerancia de las plantas en condiciones de suelos encharcados varia entre las especies (Gibbs y Leitão Filho, 1978; Kozlowski, 1984) y entre genotipos de la misma especie (Gill, 1970). Las especies tolerantes o resistentes, presentan mecanismos de adaptación que pueden ser modificaciones morfológicas, bioquímicas y/o fisiológicas, viabilizando la selección y el mejoramiento genético para esa condición del suelo. El problema del sobrehumedecimiento del suelo, que causa un situación de hipoxia, es mundial, se estima que el $6 \%$ de la superficie terrestre está ocupada por áreas anegadas o propensas a un encharcamiento temporal (Maltby, 1991). Estas áreas presentan problemas de encharcamiento temporal debido a una serie de factores: exceso de lluvias, deficiencia de drenaje, infiltración desde canales de riego e inundación por los ríos (Magalhães et al., 2000). En los suelos con estas características la difusión de los gases a la atmósfera se afecta seriamente (Armstrong et al., 1994), por otra parte la respiración aeróbica de las plantas y microorganismos reduce rápidamente los niveles de oxígeno en la solución del suelo, favoreciendo la formación de un ambiente hipóxico y anóxico (Gomes de Moraes et al., 2001), perjudicial para el buen desarrollo del sistema radical.

En Cuba, el mal drenaje afecta el 40,3\% del área agrícola cultivada, y preocupa el incremento de un 9\% estimado para los próximos 15 años, en regiones de varias provincias del país (CIGEA, 2001). En la zona del Valle del Río Cauto, se encuentra la mayor de las Ilanuras fluviales y fluviomarinas de la Isla, de una singular geodiversidad, asimila a un $10 \%$ de la población cubana, dedicada a una actividad económica bien diferenciada, donde la agricultura tiene un peso preponderante y dentro de ésta, el cultivo más extendido es la caña de azúcar (CITMA Granma, 2002). La presente investigación tuvo como objetivo determinar los afectos que provoca el sobrehumedecimiento en la profundidad del sistema radical de seis genotipos de caña cubanos, en un suelo Vertisol del Valle del Cauto en la región oriental cubana.

\section{MATERIALES Y MÉTODOS}

Los experimentos se desarrollaron en el área experimental de la Empresa Azucarera "Grito de Yara", ubicada en el municipio de Río 
Cauto, provincia Granma, Cuba, en un suelo Vertisol pélico gléyico carbonatado, según la Nueva Versión de la Clasificación Genética de los Suelos de Cuba (Hernández et al., 1999), correlacionado con la Soil Taxonomy (Soil Survey Staff, 2003) como Typic Haplustert y con el grupo Vertisoles, según la World Reference Base (Driessen et al., 2001). Los experimentos se realizaron siguiendo las Normas y Procedimientos del Programa de Mejoramiento Genético de la Caña de Azúcar en Cuba, del Instituto Nacional de Investigaciones de la Caña de Azúcar (INICA, 1987, 2002). Se plantaron dos experimentos en bloques al azar con cinco réplicas $48 \mathrm{~m}^{2}$ (4 surcos de $7.5 \mathrm{~m}$ de largo y $1.6 \mathrm{~m}$ de distancia entre surcos). Cada experimento quedó separado uno del otro a una distancia de $50 \mathrm{~m}$ para evitar la infiltración lateral del agua y en este intervalo se construyeron 10 zanjas de $0.50 \mathrm{~m}$ de profundidad separadas a $1 \mathrm{~m}$, con la misma finalidad. Por la necesidad de esclarecer las interacciones entre los genotipos y la presencia o ausencia de sobrehumedecimiento en el suelo, y debido a las dificultades con la aleatorización del factor humedecimiento del suelo, se asumió como un diseño experimental de parcelas divididas en bloques al azar con cinco réplicas. Se consideró a la presencia o ausencia del sobrehumedecimiento del suelo como parcela y a los genotipos como sub-parcelas. En la parcela sin exceso de humedad, se adoptaron todas las medidas fitotécnicas para que el factor encharcamiento fuera nulo. La otra parcela, en el mismo tipo de suelo, se concibió con la presencia del factor anegamiento. Para esto se preparó un dique de $50 \mathrm{~cm}$ de altura bordeando a la parcela, para evitar la escorrentía libre del agua y se aplicó una lámina de agua de $40 \mathrm{~cm}$ a partir de los 120 días de haber sido plantada la planta de caña y a partir de su cosecha para el primer retoño, cada 10 días tres días de anegamiento, hasta los 300 días o 10 meses, excepto cuando las precipitaciones elevaron la lámina de agua hasta los $40 \mathrm{~cm}$. Se eludieron los primeros 120 días desde la plantación, por ser la etapa más susceptible al sobrehumedecimiento (Van Dillewijn, 1951). Desde el momento de la plantación hasta los 120 días, a ambos experimentos se les aplicó riego por gravedad con una norma neta parcial de $237 \mathrm{~m}^{3} \mathrm{ha}^{-1}$, cada 12 días, para garantizar homogeneidad en la brotación y el ahijamiento, manteniéndose el riego en el experimento no sobrehumedecido hasta los 10 meses.

Los genotipos utilizados, cuyos progenitores se encuentran entre paréntesis fueron C86-12 (Desconocidos), C86-456 (PR980 x Ja60-5), C90-317 (C187-68 x B6368), C87-51 (Co281 x POJ2878), C1051-73 (B42231 x C431-62) y C120-78 (Co421 x C87-51) (Bernal et al., 1997; Jorge et al., 2004). Las mismas son fruto del programa de mejora cubano para la obtención de variedades de caña de azúcar tolerantes a condiciones de sobrehumedecimiento, el cual adolece de estudios morfofisiológicos que profundicen en el conocimiento de los mecanismos de adaptación. Se seleccionaron además tres testigos comerciales que no responden adecuadamente a estas condiciones (C105173, C87-51 y C120-78). Se seleccionó como material de plantación entrenudos de tres yemas procedentes del Banco de Semilla Básica, con tratamiento térmico y químico. Se plantaron dos entrenudos en el fondo del surco, de forma corrida, garantizándose una población superior al 98\% en el experimento.

La plantación se realizó en el mes de enero del 2002. Las labores fitotécnicas realizadas a los experimentos, aparte del manejo del agua de riego abordada anteriormente, fueron seis limpias y un aporque, todos de forma manual, mientras que la fertilización fue la establecida por el Servicio de Recomendaciones de Fertilizantes y Enmiendas (SERFE) del Instituto Nacional de Investigaciones de la Caña de 
Azúcar. Los ciclos de cosecha evaluados fueron caña planta y primer retoño. Se estudió la profundidad que alcanza el $70 \%$ del peso freso del sistema radical por su relación estrecha con las partes aéreas de la planta y por ser el órgano que está directamente más influenciado por deficiencia de oxígeno debido a excesos de agua en el suelo. El muestreo y estudio del sistema radical se realizó por variedad a los 10 meses de edad, en ambos experimentos y ciclos de cosecha, tomando en consideración la técnica del monolito, descrita, por Kolensnikov (1971), asumiendo las profundidades de muestreo recomendadas para caña de azúcar por Krautman (1959). Con la sumatoria de los pesos frescos de las raíces cada 0,10 m., se procedió a la determinación de las profundidades que alcanzan el $70 \%$ del peso total de raíces, desde cero hasta un metro. Las excavaciones se realizaron en tres réplicas diferentes, para un total de 18 muestreos por cada condición de humedad del suelo. Como atributo de tolerancia al estrés se estimó la Intensidad del Estrés (IE) o pérdida relativa de la variable seleccionada (profundidad del sistema radical) de Fisher y Maurer (1978).

Ante todo se verificó si la base de datos primaria cumplía con las premisas del análisis de varianza: el ajuste a la distribución normal, a través de la prueba de Kolmogorov-Smirnov y la de homogeneidad de las varianzas, aplicando el Test de Bartlett (Pr£ 0.05). El análisis de varianza por el modelo de efectos fijos se empleó para ambos ciclos de cosecha, en la variable, profundidad del sistema radical. Para la comparación múltiple de medias se utilizó la prueba de Tukey, $(0,05)$. Se muestran las medias de la interacción o de los factores independientes, con sus respectivos errores estándar, cuyas unidades de medida coinciden con sus variables correspondientes. Se empleó la prueba de $t$ para conocer si las afectaciones que provoca el estrés por sobrehumedecimiento del suelo fueron significativas, al comparar individualmente cada genotipo en las dos condiciones de humedad presentes en el suelo para la profundidad del sistema radical. Se muestra el nivel de significación entre las medias de cada variedad en cada condición, siendo el punto de partida para la estimación de la Intensidad del Estrés (IE) o pérdida relativa de la variable en estudio. En todas las pruebas secuenciales, los valores de $p$ fueron corregidos según el ajuste de Bonferroni (Rice, 1989), atendiendo a la modificación propuesta por Chandler (1995). Para el procesamiento automatizado de los datos se emplearon los paquetes estadísticos SAS (2000).

\section{RESULTADOS Y DISCUSIÓN}

El desarrollo que alcanzó el 70\% del peso fresco del sistema radical en profundidad se muestra en la figura 1. Se encontró ausencia de interacción entre los factores en ambos ciclos de cosecha, con reducciones estadísticamente significativas en el sistema radical provocadas por la Intensidad del Estrés, debido a excesos de agua en el suelo, con valores de $17 \%$ en caña planta y del $11 \%$ en el primer retoño, respectivamente (Figuras $1-a_{1}$ y $\left.1-b_{1}\right)$, de igual manera la respuesta de los genotipos estuvo marcada por su diferenciado comportamiento estadístico (Figuras $1-a_{2}$ y $1-b_{2}$ ). Basado en lo anterior se puede afirmar que el sistema radical de los genotipos estudiados no penetró a la misma profundidad en ambas condiciones de humedecimiento del suelo, con diferencias entre variedades. Igualmente, el $70 \%$ del peso del sistema radical en caña planta como promedio se alcanzó a los treinta centímetros en condiciones de no sobrehumedecimiento, superando a la variante sobrehumedecida para esta profundidad. Esta zona radical ha sido descrita por varios autores, Reynoso (1963), Humbert (1965) y Eppik (1972), como 
la más activa y de máxima concentración de raíces donde se realizan las labores de cultivo. Tercero, el sistema radical de las variedades C86-12, C86-456 y C90-317, penetró a mayor profundidad que el resto; con valores intermedios se comportó la C87-51, siendo el sistema radical más superficial para las variedades C1051-73 y C120-78 en las dos situaciones de humedad del suelo. La fundamentación anterior es válida para el primer retoño, pero a una menor escala (Figura 1).

La reducción del sistema radical como consecuencia del exceso de humedad en el suelo, aunque con diferenciación varietal, trae consigo que aquellas variedades con sistema radical más superficial, se vean limitadas para la absorción de nutrientes y agua, lo que las limita para enfrentar períodos de escasas precipitaciones dada la distribución superficial de su sistema radical, agravado por el agrietamiento de los suelos plásticos, que comenzó a ser visible en esta investigación a los 12 meses, lo que provoca ruptura de las raíces, principalmente las más superficiales y la planta comienza a padecer estrés por déficit hídrico debido a esas circunstancias. En esta misma zona geográfica, Cid (1992), encontró que el agrietamiento fue más intenso, donde hubo mayor humedad del suelo, lo que provocó que estos suelos hagan un uso ineficiente tanto del agua de riego, como del agua de Iluvia y nutrientes, al evacuar por esta vía más de $94 \%$ del agua, ya que la gran mayoría del agua que entra a las grietas es evacuada rápidamente sin que la planta pueda hacer uso de la misma, fenómeno denominado por Dekker y Bouma (1978), como flujo preferencial o corto circuito. Estas grietas pueden alcanzar un ancho en la superficie del suelo, que puede exceder los $15 \mathrm{~cm}$ y una profundidad en el suelo con valores cercanos a1

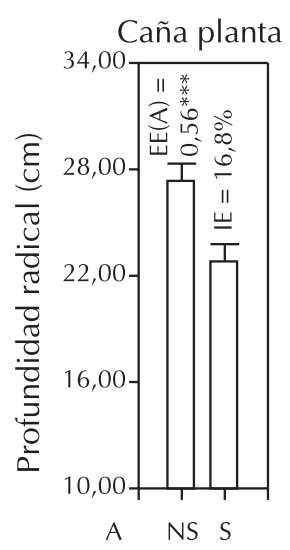

Hum. suelo a2

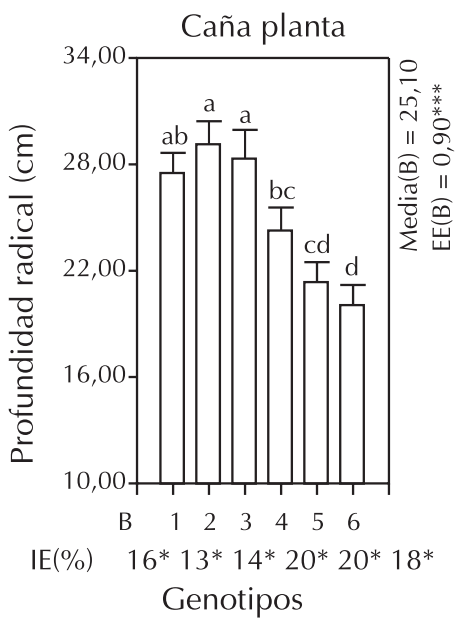

b1

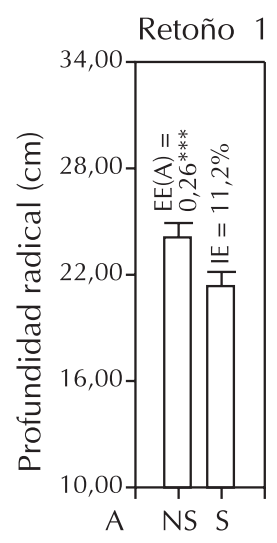

Hum. del suelo b2

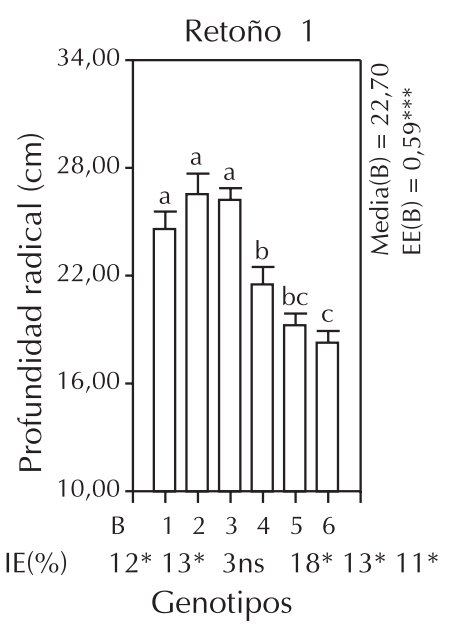

Figura 1: Comportamiento de la profundidad que alcanzó el 70\% del peso del sistema radical por ciclos de cosecha independientes, (1-a1 y 1-a2) caña planta sin interacción estadística entre los factores, (1-b1 y 1 b2) primer retoño sin interacción estadística entre los factores. Factor A, niveles de humedad del suelo; NS, no sobrehumedecido y S, sobrehumedecido. Factor B, genotipos; C86-12(1); C86-456(2); C90-317(3); C8751(4); C1051-73(5) y C120-78(6). Se incluye para cada genotipo la Intensidad del Estrés (IE) o pérdida relativa de la profundidad del sistema radical y su significación.

Nota: Letras diferentes indican diferencias significativas para p d" 0.05, empleando Tukey. $*, * * y^{* * *}$, indican diferencias significativas para $p=0.05,0.01$ y 0.001 respectivamente, empleando $t$ de student. 
a los $200 \mathrm{~cm}$ en perfiles profundos, aunque se estrechan a medida que se avanza en profundidad (Blokhuis et al., 1969). Humbert (1965), citó investigaciones en la isla de Java, que demostraron en caña planta de siete meses de edad, por lo menos el $82 \%$ de las raíces se encontraban en un horizonte de $40 \mathrm{~cm}$ de profundidad. Van Dillewijn (1951), realizó un estudio detallado de la distribución radical de la caña, por niveles de profundidad y encontró que en los primeros $40 \mathrm{~cm}$ se acumulaba el $85 \%$ de las raíces y en los primeros $60 \mathrm{~cm}$ el 94\%. En Cuba, Hernández (1981) concluyó que el peso fundamental de las raíces se encontró entre 20 y $30 \mathrm{~cm}$ de profundidad, cuando empleó como técnica de riego el goteo, en un suelo rojo. Para vertisoles, Fonseca y Lamelas (1981), destacaron la presencia de una capa impermeable entre 35 y $50 \mathrm{~cm}$ de profundidad, que dificultó el desarrollo radical en profundidad. Feldman (1984), demostró que las raíces que se desarrollan en suelos anegados son más cortas, rectas y ramificadas, que aquellas que prosperan en suelos bien aireados. En Colombia, para caña de azúcar Amaya et al. (1995), enfatizaron la importancia del sistema radical como órgano de anclaje y el medio para la absorción de nutrientes y agua del suelo, insistieron que la cantidad y la longitud de las raíces permanentes dependen de las variedades; sin embargo, existen factores ambientales como el tipo de suelo y la humedad que influyen en sus características, citaron a modo de ejemplo, lo que sucede en los suelos arcillosos sobrehumedecidos, donde se puede reducir la longitud de las raíces. Sobre lo anterior, Chávez (1999), asegura que existe respuesta varietal del sistema radical de la caña de azúcar y destacó la presencia de gravitropismo diferencial entre clones, lo cuál es regulado en general por múltiples genes (Zobel, 1991).

Basado en lo descrito por Van Dillewijn (1951), acerca de que el desarrollo de las raíces en caña de azúcar es un carácter heredable, Morris y Tai (2004), concluyeron al evaluar 12 genotipos, que la tolerancia de la caña de azúcar a excesos de humedad, incluyó un incremento de la masa de raíces en profundidad y una reducción de su diámetro cerca de la superficie del suelo. Rodríguez et al. (2005), alcanzaron valores similares de la profundidad radical alcanzada por el $70 \%$ del peso fresco, con presencia y ausencia de sobrehumedecimiento de un Vertisol en esta misma zona geográfica cubana para la variedad C86-456 obtenida por cultivo in vitro, en un experimento que incluyó además a la bacteria Azospirillum brasilense. Pizarro (1978), describe que los suelos muy húmedos son más fríos, lo que provoca una reducción de la germinación y en el desarrollo del sistema radical.

La arquitectura del sistema radical en profundidad, es particularmente importante para la absorción de fósforo, debido a la baja movilidad de este elemento en el suelo. El movimiento del fósforo es gobernado mayoritariamente por difusión, por tanto las propias plantas contribuyen a la heterogeneidad espacial de este nutriente por agotamiento en la zona rizosférica (Tinker y Nye, 2000). En varios cultivos se ha demostrado que el desarrollo del sistema radical en profundidad de ciertos genotipos es una adaptación para la absorción de fósforo (Manske et al., 2000; Lynch y Brown, 2001; Liao et al., 2001).

La reducción de la biomasa del sistema radical de la caña de azúcar, debido al sobrehumedecimiento del suelo, puede estar relacionado también con la acumulación de sustancias tóxicas en las raíces, las que inhiben la absorción de nutrientes minerales y causan un descenso en las disponibilidades de estos elementos en el suelo. Tales modificaciones se deben a alteraciones en el estado de oxidación, variaciones en el $\mathrm{pH}$, 
inhibición de los mecanismos de absorción en virtud de una carga energética baja de las membranas y por otro lado, a la propia reducción de la superficie de absorción provocada por la muerte de las raíces y la pérdida de la biomasa del sistema radical (Drew, 1997, De Carvalho e Ishida, 2002). En igual medida por efecto del exceso de agua en el suelo, ocurre una disminución de la absorción de agua, tanto por la reducción de la superficie total del sistema radical, como consecuencia de su muerte, como por el aumento de la resistencia al flujo de agua (Kozlowski, 1997).

\section{CONCLUSIONES}

- Excesos de agua en los Vertisoles reducen el desarrollo del sistema radical en profundidad de la caña de azúcar.

- El sistema radical de los genotipos C86-456, C90-469 y C86-12 penetra a mayor profundidad en los suelos sobrehumedecidos, lo que demuestra la existencia de una marcada diferencia varietal.

- El sistema radical del primer retoño es más superficial que el de caña planta.

\section{BIBLIOGRAFÍA}

Amaya, A.; Cock, J.; Del Pilar, A. e Irving, J. 1995. Biología. En: Casslett, C.; Aguas, J. y Isaacs, C. (Ed.). El Cultivo de la Caña en la Zona Azucarera de Colombia. Centro de Investigaciones de la Caña de Azúcar de Colombia (CENICAÑA), Cali, 412pp.

Armstrong, W.; Rändle, R. y Jackson, M. 1994. Mechanisms of flood tolerance in plants. Acta Botanica Neerlandica 43(4): 51-58

Bernal, N.; Morales, F.; Gálvez, G. y Jorge, I. 1997. Variedades de Caña de Azúcar. Uso y Manejo. Instituto Nacional de Investigaciones de la Caña de Azúcar, La Habana, IMAGO, 99 pp.

Blokhuis, W.; Pape, T. y Slager, S. 1969. Morphology and distribution of pedogenic carbonate in some Vertisols of the Sudan. Geoderma 2:173-200

Chandler, C. 1995. Practical considerations in the use of simultaneous inference for multiple test. Animal Behaviour 49:524-527
Chávez, M. 1999. Nutrición y fertilización de la caña de azúcar en Costa Rica. Dirección de Investigación y Extensión de la Caña de Azúcar (LAICA-DIECA), San José, 43 pp.

Cid, L. 1992. Efectos de los fenómenos de contracción-dilatación sobre las transferencias de agua y aire en suelos con arcillas dilatables. Tesis Ph. D., Instituto de Investigaciones de Riego y Drenaje, La Habana

CIGEA. 2001. Síntesis del diagnóstico de la desertificación y la sequía en Cuba. Caracterización del archipiélago cubano. Centro de Información, Gestión y Educación Ambiental, La Habana, 23pp.

Citma Granma. 2002. Cuenca hidrográfica del Río Cauto. Diagnóstico ambiental integral para un manejo sostenible. CIGEA, Bayamo, 52pp.

Crane, J. y Davies, F. 1988. Periodic and seasonal flooding effects on survival, 
growth, and stomatal conductance of young Rabbiteye Blueberry plants. Journal of the American Society for Horticultural Science 113(4):488-493

Crawford, R. y Tyler, T. 1969. Organic acid metabolism in relation to flooding tolerance in roots. Journal of Ecology 57(1):237

De Carvalho, C. e Ishida, F. 2002. Responses of young pijuayo plants (Bactris gasipaes Kunth) to flooding. Pesquisa Agropecuária Brasileira 37(9):1231-1237

Dekker, L. y Bouma, J. 1978. The influence of improved drainage on vertical hydraulic conductivity of some heavy clay soils (in Dutch). Rapport 1416: Stichting voor

Drew, M. 1997. Oxygen deficiency and metabolism: injury and acclimation under hypoxia and anoxia. Annual Review of Plant Physiology and Plant Molecular Biology 48: 223-250

Driessen, P.; Deckers, J.; Sparargaren, O. y Nachtergaele, F. 2001. Lecture Notes on the Major Soils of the World. World Soil Resources Reports 94, FAO, Rome, 334pp.

Eppik, L. 1972. Las bases del servicio de control de humedad y sus aplicaciones prácticas. CECOAAP, Lima, 25pp.

Fisher, R. y Maurer, R. 1978. Drought resistance in spring wheat cultivars. I. Grain yield responses. Australian Journal of Agricultural Research 29:897-917

Fonseca, J. y Lamelas, C. 1981. Experiencias de régimen de riego controlado y organización del riego. Empresa Cañera Rubén Martínez Villena,
Instituto de Investigaciones de Riego y Drenaje, La Habana, 9pp.

Feldman, L. 1984. Regulation of root development. Annual Review of Plant Physiology 35:223-242

Gibbs, P. y Leitão Filho, H. 1978. Floristic composition of na área of gallery Forest near Mogi-Iguaçu, state of São Pablo, S. E. Brazil. Revista Brasileira de Botânica, São Paulo 1(2):151-156

Gill, C. 1970. The flooding tolerance of woody species, a review. Forestry Abstracts, Farnham Royal 31:671-688

Gomes de Moraes, M.; Donizeti Alves, J.; Mota de Oliveira, L.; Vitorino, P. y Murad, M. 2001. Caractericâo do crescimento e da atividae das desidrogenases alcohólica e lática em seis espécies herbáceas sob condicôes de hipoxia. Ciencia y Agrotecnología, 25(1): 86-95

Hall, T. F. y Smith, G. E. 1955. Effects of flooding on woody plants, west sandy dewatering project, Kentucky Reservoir. Journal of Forestry 53(4):281-235.

Hernández, A.; Pérez, J.; Bosch, D. y Rivero, L. 1999. Nueva versión de la clasificación genética de los suelos de Cuba. Agroinfor, La Habana, 64pp.

Humbert, R. 1965. El cultivo de la caña de azúcar. Universitaria, La Habana, 785pp.

Hernández, A. 1981. Determinación del régimen de riego de la caña de azúcar para suelos rojos de la provincia de La Habana. IV Foro Científico de la Academia de Ciencias de Cuba, La Habana 
INICA (Instituto Nacional de Investigaciones de la Caña de Azúcar). 1987. Normas y Procedimientos del Mejoramiento Genético de la Caña de Azúcar en Cuba. MINAZ, La Habana, 147pp.

INICA (Instituto Nacional de Investigaciones de la Caña de Azúcar). 2002. Normas y Procedimientos del Programa de Mejoramiento Genético de la Caña de Azúcar en Cuba. Boletín No. 1 Cuba \& Caña-INICA, La Habana, 315pp.

Jackson, M. y Drew, M. 1984. Effects of flooding on growth and metabolism of herbaceous plants. En: Koslowski, T. (Ed.). Flooding and Plant Growth, Academic Press, London, p47-128

Jorge, H.; Morales, F.; Jorge, I. y García, H. 2004. Catálogo de nuevas variedades de caña de azúcar. Publicaciones INICA, La Habana, 105pp.

Kawase, M. 1987. Annatomical and morphological adaptation of plants to waterlogging. HortScience 16(1):30-34

Kolensnikov, V. 1971. The root system of fruit plant. MIR, Moscow, 269pp.

Kozlowski, T. 1984. Response of woody plants to flooding. En: Koslowski, T. (Ed.). Flooding and Plant Growth, Academic Press, London, p129-163

Krautman, S. 1959. Observacoes do sistema radicular da Cana IANE-C46117 en solo de Baixada (Varzea). Boletim Técnico do Instituto Agronomico do Norte (6):2531

Kozlowski, T. 1997. Responses of woody plants to flooding and salinity. Tree Physiology Monograph Victoria, 1:1-29
Liao, H.; Rubio, G.; Yan, X.; Cao, A.; Brown, K. y Lynch, J. 2001. Effect of phosphorus availability on basal root shallowness in common bean. Plant and Soil 232:69-79

Lynch, J. y Brown, K. 2001. Topsoil foraging, an architectural adaptations of plants to low phosphorus availability. Plant and Soil 237:225-237

Magalhães, P.; F. Durães; Patrícia de F. Vitorino; J. Donozeti Alves y E. Gama. 2000. Adaptación del maíz a condiciones de estrés por encharcamiento. http:// www.turipana.org.co/estres_maiz.htm. [Accedido: 05 - 25 -2007]

Maltby, E. 1991. Wetlands their status and role in the biosphere. In: JACKSON, M.; Davies, D.; Lambers, H. Plant life under oxygen deprivation. Ecology, physiology and biochemistry. SPB Academic, The Hague, p3-21

Manske, G.; Ortíz - Monasteri, J.; Van Ginkel, M.; González, R.; Rajaram, S.; Molina, E. y Viek, P. 2000. Traits associated with improved P-uptake efficiency in CIMMYT's semiwarf spring bread wheat grown on an acid Andisol in México. Plant Soil 221:189-204

Morris, D. y Tai, P. 2004. Water table effects on sugarcane root and shoot development. American Society of Sugar Cane Technologists 24:41-59

Pizarro, F. 1978. Drenaje agrícola y recuperación de suelos salinos. Editora Agrícola S. A., Madrid, 521pp.

Reynoso, A. 1963. Ensayo sobre el cultivo de la caña de azúcar. Editorial Nacional de Cuba, La Habana, 520pp. 
Rice, W. 1989. Analysing tables of statistical test. Evolution 43:223-225

Rodríguez, S.; Triana, R.; Romero, J.; Olivera, W.; Pérez, J.; Expósito, I.; Gonzáles, A.; Pérez, Y.; López, G.; López, S.; Loddo, Z.; Cabrera, A.y Castillo, A. 2005. Efecto de Azospirillum brasilense sobre la cosecha y el desarrollo radical de plantas de caña de azúcar (Saccharum spp.), variedad C86-456 obtenidas por cultivo in vitro, en condiciones normales y bajo sobrehumedecimiento del suelo. Biotecnología Vegetal 5(1):39-43

Soil Survey Staff. 2003. Claves para la Taxonomía de Suelos. Colegio de Postgraduados, Chapingo, 306pp.
SAS Institute. 2000. Statistical Analysis System. SAS Institute. User's Guide. Version 8.0. SAS Institute, Inc., Cary, North Carolina, 3884 pp.

Tinker, P. y Nye, P. 2000. Solute movement in the rhizosphere. Oxford University Press, New York, 280pp.

Van Dillewijn C. 1951. Botánica de la caña de azúcar. Edición Revolucionaria. Instituto cubano del libro. Segunda edición. La Habana, 460 pp.

Zobel, R. 1991. Genetic control of root system. En: Waisel, Y.; Eshel, A. y Kafkafi, U. (Ed.). Plant Roots: the hidden half. Marcel Dekker, New York, 27-38 pp. 